\title{
In-Situ Characterization of the Evolution of Defects in AIGaN/GaN HEMTs in the On-state and Off-state condition
}

\author{
A. Lang $^{1}$, H. Ghassemi ${ }^{1}$, D. Meyer ${ }^{2}$ and M. L. Taheri ${ }^{1}$ \\ 1 . Department of Materials Science and Engineering, Drexel University, Philadelphia, PA, 19140 \\ ${ }^{2}$. U.S. Naval Research Laboratory, Washington, DC 20375
}

Nitride semiconductors offer many unique and beneficial properties for new generation electronic devices [1]. GaN-based HEMTs are contenders for replace existing Si and GaAs devices in high-power RF applications. AlGaN/GaN High Election Mobility Transistors (HEMTs) are devices designed for applications where high-power and high-frequency devices are needed. AlGaN/GaN HEMTs take advantage of a two-dimensional electron gas (2DEG) that forms at the AlGaN/GaN interface to create a layer of highly mobile electrons that are easily modulated by an applied bias. Unfortunately, high-power operating conditions often result in unpredictable and catastrophic device degradation [2]. In our previous ex-situ work, we observed the formation of defects under the drain side edge of the gate during bias. The amount of defects present increased with bias duration and this was related to changes in $I_{D}$ and strain near the $\mathrm{AlGaN} / \mathrm{GaN}$ interface [3]. However, the formation mechanism of these defects has not been fully investigated as a function of operating time. As such, quantitative analysis on the evolution of defects is needed to further understand device failure mechanisms.

In this work we employed in-situ electrical biasing using TEM to investigate AlGaN/GaN HEMT device degradation during operation. Specifically, we compare device degradation during both off- and on-state stresses. Off-state stressing causes the device to enter a pinch off state, wherein the 2DEG is depleted of mobile carriers and the device is only subject to electric field effects. While on-state stressing causes the device to experience both electric field and thermal effects induced by flowing current through the device and better represent real operating conditions. Preliminary in-situ TEM results are shown in figure 1 , the increasing concentration of defects is in agreement with our ex-situ results.

All samples were prepared using a FEI DB235 Dual-Beam FIB. Off-state in-situ biasing was performed using Protochips electrical biasing chips in a two terminal configuration and on-state in-situ biasing was performed in a custom built Hummingbird electrical biasing holder using a three terminal configuration. In-situ studies are coupled with geometric phase analysis and NanoMEGAS orientation mapping to quantify the strain evolution throughout the device. After biasing, we perform analytical-TEM measurements on the defects that arise from biasing in order to analyze the composition and structural evolution of these defects with biasing duration using HAADF and EELS. This research serves to improve the understanding of failure mechanisms of GaN HEMTs during operation and can lead to improved degradation models and better device reliability.

\section{References:}

[1] Morkoç, H., Handbook of Nitride Semiconductors and Devices, GaN-based Optical and Electronic Devices. 2008: Wiley.

[2] Joh, J., et al., Microelectronics Reliability, 51(2): p. 201-206. (2011)

Ghassemi, H et al. Journal of Applied Physics, 114(6) 064507 (2013) 
a)

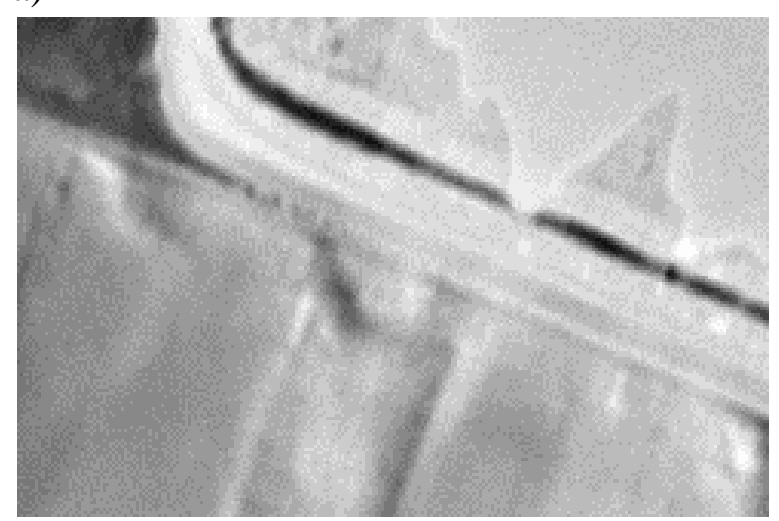

c)

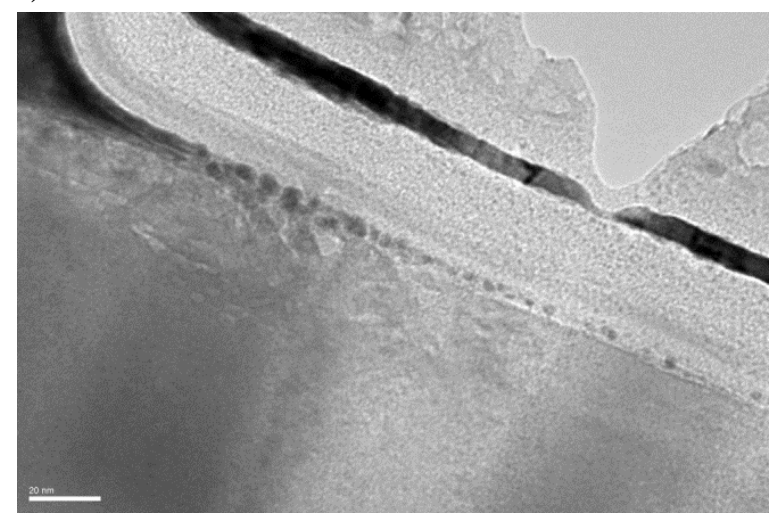

b)

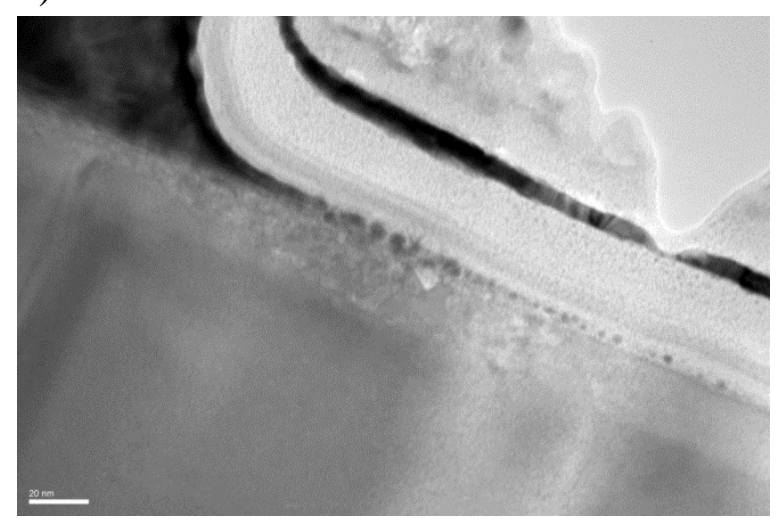

d)

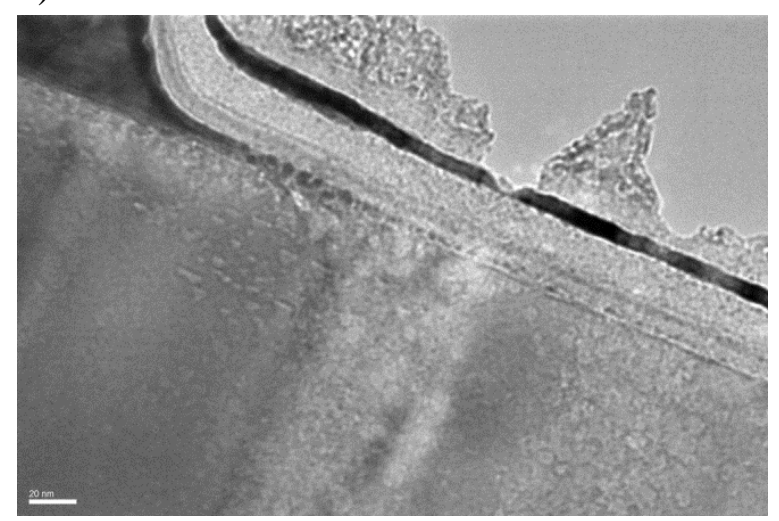

Figure 1: Four excerpts from an in-situ electrical biasing experiment on an AlGaN/GaN HEMT. The top left image, a, was taken before the experiment and shows the drain side edge of the gate, an area known to experience the highest stresses and temperatures during operation, and the next three images, $\mathrm{b}-\mathrm{d}$, detail the degradation observed in this region during the experiment. The area of degradation increases with time. The scale bar in all of these images is equal to $20 \mathrm{~nm}$. 